\title{
Welcome to the 14th volume of Nanomedicine
}

\author{
Lucy Chard*,1 \\ ${ }^{1}$ Commissioning Editor, Future Medicine, Future Science Group Ltd, London, United Kingdom \\ *Author for correspondence: I.chard@futuremedicine.com
}

First draft submitted: 11 October 2018; Accepted for publication: 12 November 2018; Published online: 14 December 2018

Keywords: authorship $\bullet$ cancer research $\bullet$ nanodiagnostics $\bullet$ Nanomedicine $\bullet$ nanotherapeutics $\bullet$ readership $\bullet$ review • top content

Welcome to the 14th volume of Nanomedicine. I would like to take this opportunity to wish all of our readers a Happy New Year. In this Foreword, I shall be taking a look back at some of the journal highlights of 2018.

Over the past year, we have seen some great strides in the development of novel work and research for nanomedical applications. This advancing field, while niche, has the potential to show a more widespread application of this technology. It has been a privilege for Nanomedicine to publish work in this fascinating field, which continues to be of the highest quality.

\section{Article highlights}

We have published a number of outstanding research articles in the journal this year. Therefore, I would like to draw your attention to some of the particular highlights of 2018.

At the time of writing our most-read research article from 2018 was entitled 'Sorafenib-loaded polymeric micelles as passive targeting therapeutic agents for hepatocellular carcinoma therapy' by $\mathrm{Su}$ et al. [1]. The research paper was dedicated to developing a better delivery system for Sorafenib to combat its low bioavailability and increase its efficacy for the treatment of hepatocellular carcinoma (HCC). Sorafenib is a multikinase inhibitor which interrupts the activity of tyrosine kinases. Sorafenib is the only clinically approved molecule-targeting drug as the standard treatment for advanced HCC, prompting the research. Su et al. successfully fabricated a biocompatible micelle that gave a number of advantages and increased the curative effects of the drug [1], showing great promise for the treatment of HCC.

This article is closely followed by another research paper: 'Nanosized carriers based on amphiphilic poly- $N$-vinyl2-pyrrolidone for intranuclear drug delivery' by Luss et al. [2], published online in April 2018. This study used polymer nanocarriers loaded with curcumin, and utilized different techniques, such as emulsification and ultrasonic dispersion, to determine different biological effects arising from the two distinct particle sizes. The work poses a new avenue for cancer therapeutics.

\section{Other article types}

Nanomedicine publishes a range of article types, meaning that we are able to highlight some particularly interesting and novel topics through reviews and editorial pieces.

One notably popular Editorial article was: 'How can 3D printing be a powerful tool in nanomedicine?' by Zhang et al. from The George Washington University (DC, USA) [3]. The article discussed the use of nanoscaled 3D-printed objects to be used in specific medical situations such as for controlled drug release, imaging and diagnostics.

Another of the most highly read articles of 2018, entitled 'Application of nanodiagnostics and nanotherapy to CNS diseases', was a review authored by He et al. [4]. The review covered the most prominent CNS diseases and the nanotherapeutic and diagnostic applications for such diseases. The authors highlighted the many advantages of

Future : Medicine 


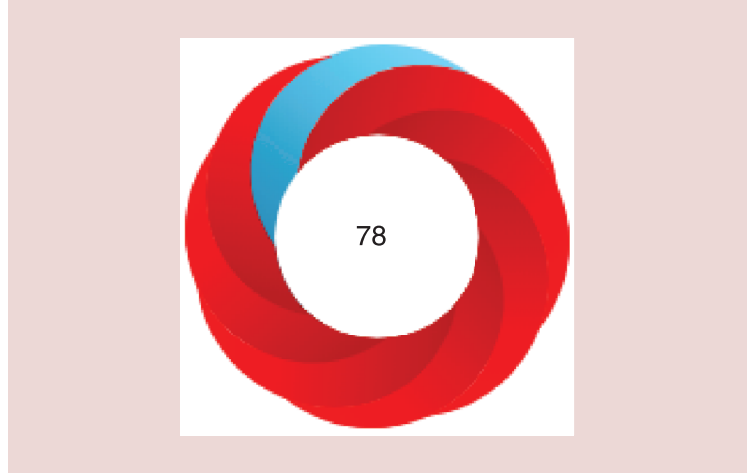

Figure 1. The colors of the wheel represent kinds of sources, a visualization of the 'Altmetric Attention Score'. Here, the red represents news outlets and the light blue represents Twitter [6].

the use of nanoparticles for overcoming challenges presented by late disease onset/detection and the blood-brain barrier.

A perspective article is an excellent way for scientists to communicate, with journals such as Nanomedicine acting as a platform for opinions to be expressed. One such perspective had a high impact in 2018: 'Are existing standard methods suitable for the evaluation of nanomedicines: some case studies' by Gioria et al. [5] The authors here challenged the testing process of nanomaterials for biomedical applications suggesting there might lie the reason for failure in clinical testing stages of some nanomaterials. They also touched on the safety aspect of medical devices and nanomaterials for in vitro use [5]. The article calls for the development of higher standards in nanomedicine to encourage a higher standard in the field for the future.

\section{Interviews}

As nanomedicine can be integrated into many different disciplines, it has been interesting to see what our authors think of the field and future directions. This year we were able to attend conferences such as the ACS National Meeting in Boston and speak to many experts in the field. It has been a pleasure to talk to the researchers and get a feel for why they are conducting their research and where they think nanomedicine has the scope to go in the future, while bringing the community together to open up a wider discussion looking toward 2019.

\section{Altmetrics}

Altmetrics is a powerful way to measure the impact of our publications. It collates information from multiple different sources on different platforms to show the way in which the research is being broadcast across the world.

The most prominent example of this from 2018 in Nanomedicine has been the research article by Jiang et al. entitled: ' $1 \alpha, 25$-dihydroxyvitamin D3-eluting nanofibrous dressings induce endogenous antimicrobial peptide expression' [6]. This has received an Altmetrics score of 78 since its publication in June. The research has been picked up by several news outlets and garnered much attention on Mendeley and social media. The article has engaged readers mainly from North America. In this study, the team collaborated across many universities throughout the USA, to form a nanofibrous wound dressing which could be used to reduce infection of surgical wound sites [6].

The Altmetrics features a useful graphic to visualize the 'Altmetric Attention Score' for a research output (Figure 1). The score is derived from an automated algorithm, and represents a weighted count of the amount of attention received for a research output [7].

\section{Geographical spread of our contributors}

As the diversity of the research community continues to grow globally, we have also seen changes in the readership of Nanomedicine from one year to the next. This past year, our content was most read by experts in Asia (37\%). North America and Europe also make up a large proportion of our readership with 19 and 14\%, respectively (Figure 2).

It is also interesting to see the proportion of authors from different countries and how this is comparable to the readership, with a higher number of authors from Asia and Europe being published (Figure 3).

Nanomedicine continues to welcome both readers and content from across the world. 
Figure 2. Proportion of readership demographics for Nanomedicine in 2018.

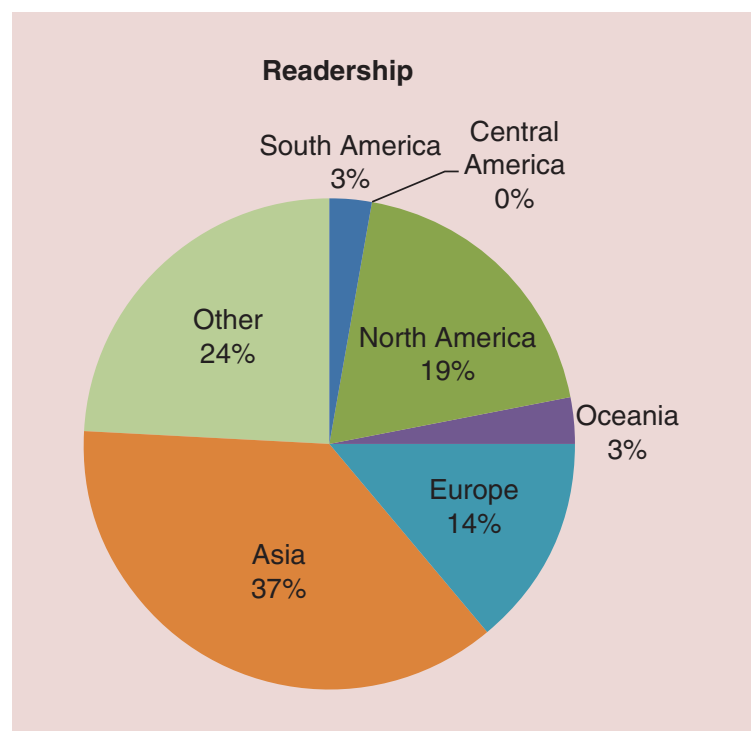

Figure 3. Proportion of authorship demographics for Nanomedicine in 2018.

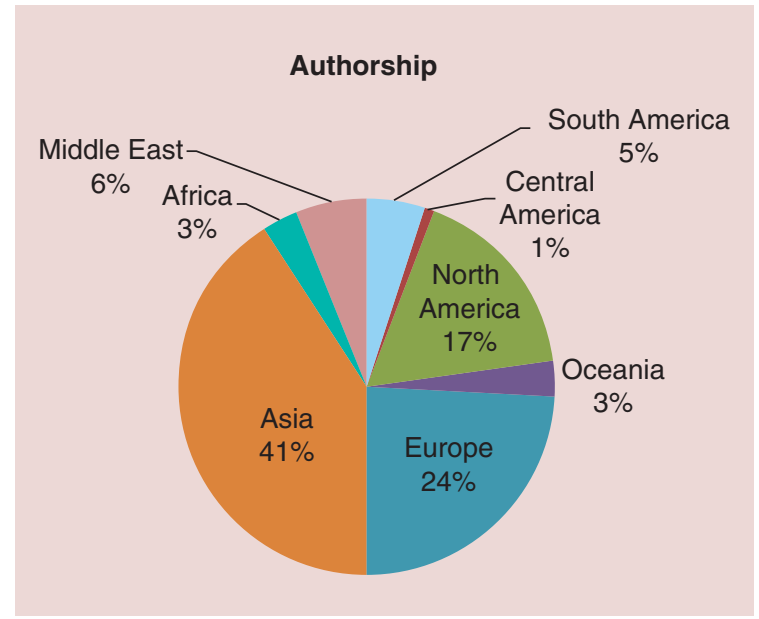

\section{Twitter}

As many of our readers will be aware, Nanomedicine has a Twitter account. We use Twitter to showcase content highlights, journal updates and key news articles from the field. With around 1500 followers this is an excellent way to feature the journal and its contents on a global platform, as well as sharing topical news as it happens. It has been a pleasure to engage with our authors and readers in a more informal setting by using Twitter and I encourage anyone on Twitter to follow us (@fsgnnm) [8].

\section{Conclusion}

I would like to take this opportunity to thank everyone that has helped with the success of Nanomedicine in 2018. We welcome unsolicited research, review and editorial article proposals, and would be delighted to hear from you if you are interested in submitting to the journal to continue on this success into 2019.

Many thanks go to the Editorial Board for continued support and to the readers for your continuing interest Nanomedicine, I look forward to collaborating with many of you over the coming year.

Financial \& competing interests disclosure

L Chard is an employee of Future Medicine Ltd. The author has no other relevant affiliations or financial involvement with any organization or entity with a financial interest in or financial conflict with the subject matter or materials discussed in the manuscript apart from those disclosed.

No writing assistance was utilized in the production of this manuscript. 


\section{References}

1. Su Y, Wang K, Li Y et al. Sorafenib-loaded polymeric micelles as passive targeting therapeutic agents for hepatocellular carcinoma therapy. Nanomedicine 13(9), 1009-1023 (2018).

2. Luss A, Kulikov P, Romme $S$ et al. Nanosized carriers based on amphiphilic poly- $N$-vinyl-2-pyrrolidone for intranuclear drug delivery. Nanomedicine 13(7), 703-715 (2018).

3. Zhu W, Webster T, Zhang L. How can 3D printing be a powerful tool in nanomedicine? Nanomedicine 13(3), 251-253 (2018).

4. Zhang W, Wang W, Yu D, Xia Z, He Z. Application of nanodiagnostics and nanotherapy to CNS diseases. Nanomedicine 13(18), 2341-2371 (2018).

5. Gioria S, Caputo F, Urbán P et al. Are existing standard methods suitable for the evaluation of nanomedicines: some case studies. Nanomedicine 13(5), 539-554 (2018).

6. Jiang J, Zhang Y, Indra A et al. 1 $\alpha, 25$-dihydroxyvitamin D3-eluting nanofibrous dressings induce endogenous antimicrobial peptide expression. Nanomedicine 13(12), 1417-1432 (2018).

7. Altmetric.com. (2018). Altmetric explorer. www.altmetric.com/explorer/outputs?journal_id\%5B\%5D=4f6fa62e3cf058f61000823a\&p ublished_after=2018-01-01\&published_before=2018-10-22\&show_details=44513235

8. Twitter.com. (2018). Nanomedicine Journal (@fsgnnm) on Twitter. https://twitter.com/fsgnnm 